\title{
Complicated gastrointestinal tuberculosis in children: case report
}

\section{Introduction}

Tuberculosis (TB) is a chronic granulomatous disease produced by Mycobacterium Tuberculosis. It has a high incidence and mortality in different parts of the world. The estimated prevalence of tuberculosis is 10.4 million of cases worldwide, with 1.8 million of annual deaths. ${ }^{1}$ India is the country with the highest number of reported cases, representing approximately $26 \%$ of the patients with tuberculosis in the world. ${ }^{2} \mathrm{~TB}$ is an important endemic disease in Peru having been reported as the country with the highest load of tuberculosis in the region of Latin America and Caribbean by the Pan American Health Organization. ${ }^{3}$

Primary TB infection usually occurs in the lungs, with the possibility to spread to other parts of the body later. From the total of patients with tuberculosis, $80 \%$ have pulmonary involvement and $20 \%$ extra-pulmonary. From this $20 \%$ the abdomen is affected in $11-$ $16 \%$, and almost half of these cases involve the gut. ${ }^{4,5}$ The abdomen involvement is not frequent in children, the incidence is approximately $10 \%$ under the age of 10 years old, with pulmonary complication in $1-5 \%$ of the cases. ${ }^{6}$

Abdominal TB usually occurs in four forms: tuberculosis lymphadenopathy, peritoneal tuberculosis, gastrointestinal tuberculosis and visceral tuberculosis of solid organs. ${ }^{2,7}$ The clinical manifestations of the abdominal tuberculosis are nonspecific and can imitate many other diseases such as Cohn's disease, causing a delay in the diagnosis and therefore may present with complications. ${ }^{8-10} \mathrm{We}$ present the case of a 6year old girl with multisystemic tuberculosis and intestinal involvement complicated by intestinal perforation.

\section{Case report}

6year-old female patient entered the emergency department with abdominal pain, reporting one month's history of diarrhoea with mucus and blood approximately 10 times per day, night fever, hiporexia and loss of weight not quantified. On examination: HR 110, RR:30 T: $38.3^{\circ} \mathrm{C}$, Sat $\mathrm{O} 2: 98 \%$, awake, breathing spontaneously, pale and with decreased subcutaneous adipose tissue. Palpable cervical lymph nodes. Abdomen: Distended, tender and with resistance to palpation. Liver palpated $4 \mathrm{~cm}$ below right costal margin. No splenomegaly.

The patient was admitted to the emergency department with the following diagnoses: Abdominal Sepsis, Chronic Diarrhea, Severe Malnutrition. She was later admitted to the department of Infectious Diseases for further investigations. During her hospitalization, she deteriorated and developed an acute surgical abdomen on day 3. An Abdominal X-ray showed a pneumoperitoneum (Figure 1), which was evaluated by the surgical service. The surgeons suggested an exploratory laparotomy. In theatre, they found multiple perforations secondary to obstructions in the ileum with mesenteric lymphadenitis. The enlarged lymph nodes appeared to contain caseous necrosis.

Peritoneal cavity lavage, intestinal resection and Ileostomy were performed. A sample of intestinal tissue was sent for smear microscopy and culture which identified Mycobacterium tuberculosis complex (MTC). The immunocromatography, also
Volume 8 Issue 3 - 2017

\author{
Verastegui S Rodrigo,' Rivera Medina Juan, ${ }^{2}$ \\ De la Cruz H Luis $^{3}$ \\ 'Division of Gastroenterology, Hepatology and Nutrition, \\ Instituto Nacional de Salud del Ni, Peru \\ 2Department of Pediatrics and Gastroenterology pediatrics, \\ Universidad Nacional Mayor de San Marcos and Universidad \\ Peruana Cayetano Heredia, Peru \\ ${ }^{3}$ Department of Pathology, Instituto Nacional de Salud del Ni, \\ Peru
}

Correspondence: Verastegui S Rodrigo, Fellow, Division of Gastroenterology, Hepatology and Nutrition, Instituto Nacional de Salud del Ni, Peru, Email Rodrigo-verasI4@hotmail.com

Received: November 02, 2017 | Published: December 28, 2017

detected the MTC and was sensitive to all the first-line anti-TB drugs. The pathology results concluded chronic granulomatous inflammation of tuberculous aetiology with Ziehl Neelsen positive results (Figures 2-4). During her evolution hepatosplenomegaly, ascites and bilateral pleural effusions were also evident. She had also a positive acid-fast bacilli smear microscopy result in stool, tracheal secretion and gastric juice. The patient had a negative purified protein derivative (PPD) skin test. The patient received anti-tuberculous treatment for 12 months (two months of rifampicin, isoniazid, pyrazinamide, ethambutol and next 10 months of rifampicin and isoniazid) with favorable evolution.

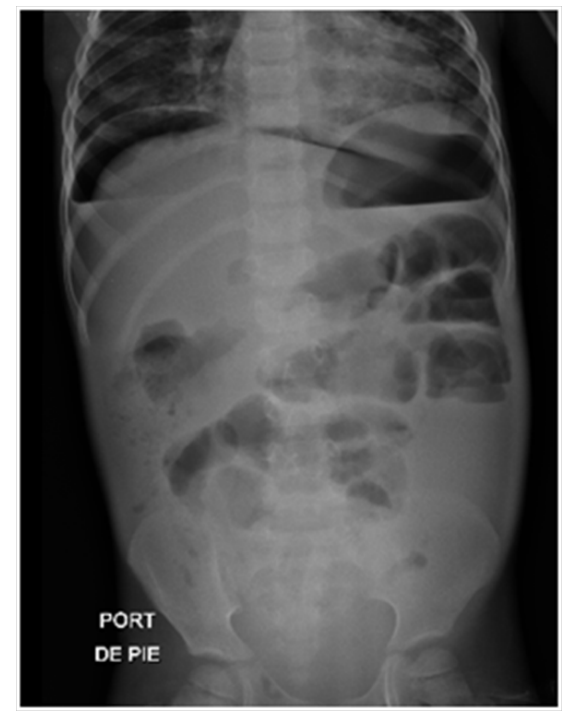

Figure I Pneumoperitoneum. 


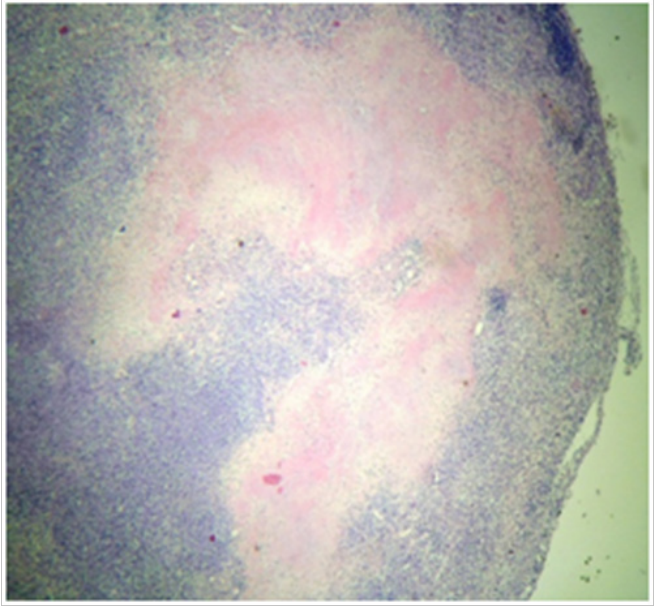

Figure 2 Caseous necrosis in the Intestine (10x magnification).

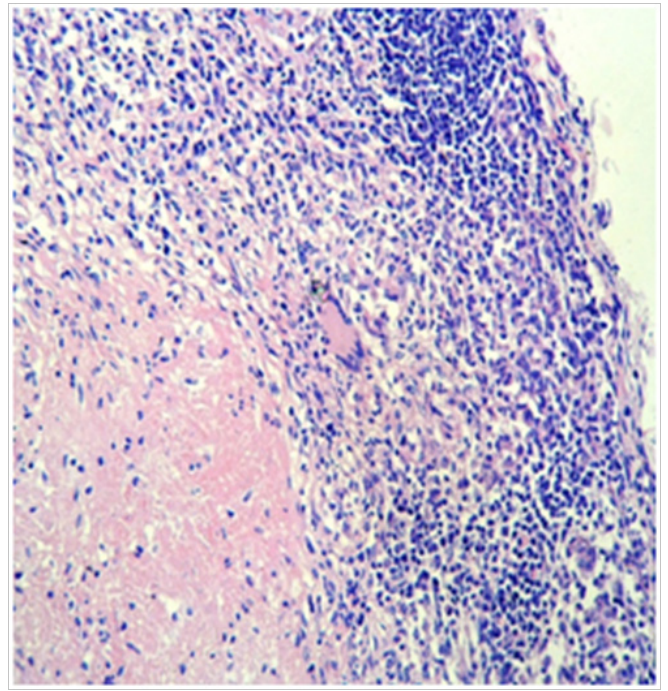

Figure 3 Granuloma formation with giant cells (40x magnification).

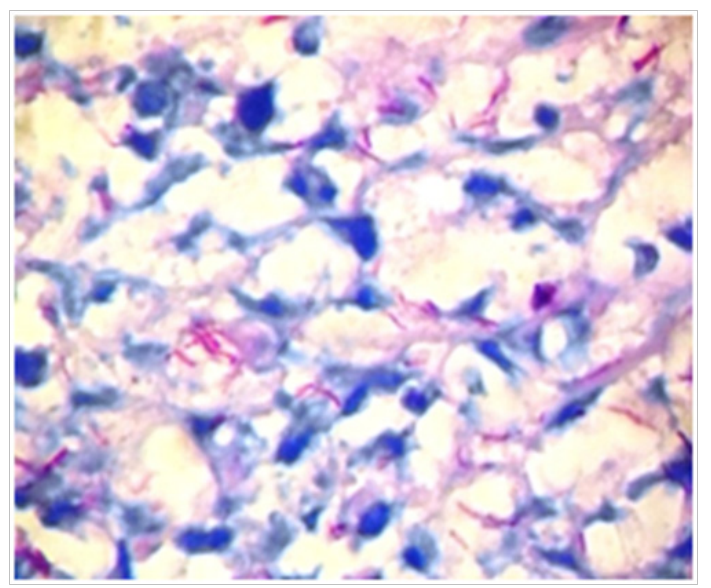

Figure 4 Mycobacterium Tuberculosis Ziehl Neelsen (I00x magnification).

\section{Discussion}

Abdominal tuberculosis is an uncommon presentation of tuberculosis, especially in children with no other debilitating disease such as cirrhosis, diabetes, and chronic renal failure with continuous ambulatory peritoneal dialysis. ${ }^{11}$ Within the various forms of presentation, intestinal involvement is one of the most frequent, and may present in a primary or secondary form. ${ }^{12}$ The primary form is caused by $M$. bovis and results from the ingestion of contaminated milk. However, this form of disease is practically not observed in industrialized countries where the elimination of infected cattle and the pasteurization of milk are efficient. The secondary form is caused by Mycobacterium tuberculosis, which originates from an extraintestinal primary focus, usually pulmonary, and extends into the intestinal tract as a result of haematogenous dissemination or after ingestion of contaminated pulmonary secretions. ${ }^{13}$ However, only $1-5 \%$ of paediatric cases of pulmonary tuberculosis complicated by abdominal infection are reported. ${ }^{14}$

A report of autopsy cases of children with tuberculosis shows that the incidence could be much greater. In this study, intestinal infection was found in $37.5 \%$ of cases, most of which were diagnosed with lung injury, the main cause of hospital admission and death. ${ }^{15}$ In the presented patient, pulmonary involvement with pleural effusion and positive Koch's bacillus in the tracheal aspirate was evidenced. Possibly this was the origin of the dissemination of Mycobacterium Tuberculosis to the intestine. In the pathogenesis of abdominal disease, subsequent to the entrance of the bacillus into the digestive system, it may invade the intestinal submucosa where it induces inflammatory changes that, when progressing, produce mucosal ulceration, caseous necrosis, decreased intestinal lumen or intestinal perforation. ${ }^{16,17}$ In patients with intestinal tuberculosis, the presentation may be acute or subacute/chronic. In the acute type, the patient presents with acute peritonitis, intestinal obstruction and/or intestinal perforation.

In the case of a subacute presentation, there may be subacute intestinal obstruction, alternation of constipation and diarrhea, palpable abdominal mass, chronic abdominal pain and weight loss. ${ }^{18}$ The reported patient had a clinical picture compatible with subacute presentation with abdominal pain and chronic diarrhea, which due to the diagnostic delay was complicated by multiple intestinal perforations at the ileocecal level that required surgical intervention.

The reported incidence of perforation in adults and children varies from 0 to $11 \%$ and is very rare in children, however due to the presentation of the disease with nonspecific clinical manifestations, the diagnosis is usually delayed and therefore allows the development of complications and increase of mortality. ${ }^{19}$ In view of the diagnostic suspicion of intestinal tuberculosis in the patient, a sample of intestinal tissue was sent for staining with Ziehl Neelsen and Culture that showed the bacillus, Mycobacterium tuberculosis. In the treatment of all patients with abdominal tuberculosis, a standard complete course of antituberculosis therapy should be administered. There is still a debate over the duration of treatment. Treatment has been reported to be successful when administered for 12-18months but other authors have reported that a duration of 6months is sufficient. Our patient received antituberculous therapy for 1year with favorable evolution. ${ }^{20,21}$

\section{Acknowledgements}

None.

\section{Conflicts of interest}

The authors declare that there are no conflicts of interest.

\section{Funding}

None. 


\section{References}

1. World Health Organization (WHO). Tuberculosis. Global Tuberculosis Report. 2016.

2. Debi U.Abdominal tuberculosis. World $J$ Gastroenterol. 2014;20(40):14831-14840.

3. Organización Panamericana de la Salud. La tuberculosis en la Región de las Américas, Informe Regional 2013. Washington DC, USA. 2014.

4. Flemming GM, Oberschmid B, Siebolts U, et al.Abdominal tuberculosis in children and adolescents: to this day a diagnostic challenge. Klin Padiatr. 2013;225(1):47-49.

5. Reto Valiente L, Pichilingue Reto C, Pichilingue Prieto O, et al.Tuberculosis abdominal en niños y adolescentes. Un desafío diagnóstico. Rev Gastroenterol Peru. 2015;35(4):318-322.

6. Lin YS, Huang YC, Lin TY. Abdominal Tuberculosis in Children: A Diagnostic Challenge. J Microbiol Immunol Infect. 2010;43(3):188-193.

7. Barman B, Nongpiur A, Bora K, et al. Clinical and laboratory presentation of abdominal tuberculosis in Shillong, Meghalaya: Experience from Northeast India. 2017;8(3):134-138.

8. Awasthi S, Saxena M, Ahmad F, et al. Abdominal tuberculosis: A diagnostic dilemma. J Clin Diagn Res. 2015;9:EC01-EC03.

9. Almadi MA, Ghosh S, Aljebreen AM. Differentiating intestinal tuberculosis from Crohn's disease: a diagnostic challenge. Am J Gastroenterol. 2009;104(4):1003-1012.

10. Makharia GK, Srivastava S, Das P, et al. Clinical, endoscopic, and histological differentiations between Crohn's disease and intestinal tuberculosis. Am J Gastroenterol. 2010;105(3):642-651.

11. Lazarus AA, Thilagar B. Abdominal tuberculosis. Dis Mon. 2007;53:32-38.
12. Cruz AT, Starke JR. Clinical manifestation of tuberculosis in children. Paediatr Resp Rev. 2007;8:107-117.

13. Ridaura-Sanz, Eduardo López-Corella, Ruy Lopez-Ridaura. Intestinal/ Peritoneal Tuberculosis in Children: An Analysis of Autopsy Cases," Tuberculosis Research and Treatment 230814. 2012.

14. Al-Fadel SM, Al-Quorain A, Larbi E, et al. Tuberculous peritonitis in children: report of two cases and literature review. $J$ Pediatr Gastroenterol Nutr. 1997;24:222-225.

15. Ridaura-Sanz C, López-Corella E, Lopez-Ridaura R. Intestinal/ peritoneal tuberculosis in children: an analysis of autopsy cases. Tuberc Res Treat. 2012;2012:230814.

16. Horvath KD, Whelan RL. Intestinal tuberculosis: Return of an old disease. Am J Gastroenterol. 1998;93(5):692-696.

17. McGee GS, Williams LF, Potts J, et al. Gastrointestinal tuberculosis; resurgence of an old pathogen. Am Surg. 1989;55:16-20.

18. Bilal Mirza, Lubna Ijaz, Muhammad Saleem, et al. Surgical aspects of intestinal tuberculosis in children: Our experience. African Journal of Paediatric Surgery. 2011; 8(2) :185-189.

19. P Martínez Tirado, Hierro Ruiz ML, Garcia RM, et al. Tuberculosis intestinal.Un reto diagnóstico. Gastroenterol Hepatol. 2003;26(6):351-354.

20. DN Sarkara, R Aminb, H Mohammed, et al. Treatment Outcome in Patients of Abdominal Tuberculosis receiving Antitubercular Chemotherapy according to National Tuberculosis Guideline of Bangladesh. Journal of Bangladesh College of Physicians and Surgeons. 2016;34(2).

21. Schofield PF. Abdominal tuberculosis. Gut. 1985:27(8):990-991. 